\title{
Sु \\ Complex magnetism of iron monolayers on hexagonal transition metal surfaces from first principles
}

\author{
B. Hardrat, ${ }^{1}$ A. Al-Zubi, ${ }^{2}$ P. Ferriani, ${ }^{1}$ S. Blügel,${ }^{2}$ G. Bihlmayer, ${ }^{2}$ and S. Heinze ${ }^{1, *}$ \\ ${ }^{1}$ Institute of Applied Physics, University of Hamburg, Jungiusstrasse 11, 20355 Hamburg, Germany \\ ${ }^{2}$ Institut für Festkörperforschung (IFF) and Institute for Advanced Simulation (IAS), Forschungszentrum Jülich, \\ D-52425 Jülich, Germany
}

(Received 3 November 2008; revised manuscript received 22 January 2009; published 11 March 2009)

\begin{abstract}
Using first-principles calculations, we demonstrate that an Fe monolayer can assume very different magnetic phases on hcp (0001) and fcc (111) surfaces of $4 d$ - and $5 d$-transition metals. Due to the substrates' $d$-band filling, the nearest-neighbor exchange coupling of Fe changes gradually from antiferromagnetic (AFM) for Fe films on $\mathrm{Tc}, \mathrm{Re}, \mathrm{Ru}$, and $\mathrm{Os}$ to ferromagnetic on $\mathrm{Rh}$, Ir, Pd, and Pt. In combination with the topological frustration on the triangular lattice of these surfaces the AFM coupling results in a $120^{\circ}$ Néel structure for $\mathrm{Fe}$ on Re and Ru and an unexpected double-row-wise AFM structure on Rh, which is a superposition of left- and right-rotating $90^{\circ}$ spin spirals.
\end{abstract}

DOI: 10.1103/PhysRevB.79.094411

PACS number(s): 75.70.Ak, 71.15.Mb

Triggered by the discovery of the giant-magnetoresistance effect and the demand to realize spintronic device concepts, ${ }^{1}$ magnetic nanostructures on surfaces have been a focus of experimental and theoretical research for more than 20 years now. In particular, there has been a tremendous effort to grow ultrathin transition-metal films on metal surfaces and to characterize and explain their magnetic properties. It is now generally believed that these structurally simple systems are well understood and more complex nanostructures such as atomic chains, clusters, or molecules on surfaces have moved into the spotlight of today's research. ${ }^{2-7}$

Therefore, it came as a big surprise when it was experimentally shown that the prototypical ferromagnet $\mathrm{Fe}$ becomes a two-dimensional (2D) antiferromagnet on the $\mathrm{W}(001)$ surface. ${ }^{8}$ Combining spin-polarized scanning tunneling microscopy and first-principles calculations, it has been further demonstrated that complex magnetic order can be obtained even in single monolayer (ML) magnetic films on nonmagnetic substrates. For example, recently a spin-spiral state was discovered for a Mn ML on W(110) (Ref. 9) and for a Mn ML on W(001) (Ref. 10) and a nanoscale magnetic structure was found for an Fe ML on $\operatorname{Ir}(111) .{ }^{11}$ Surfaces of $4 d$ - and $5 d$-transition metals (TMs) such as $\mathrm{W}, \mathrm{Re}, \mathrm{Ru}$, or Ir have been particularly attractive from an experimental point of view as ultrathin $3 d$-TM films can often be grown pseudomorphically and without intermixing. ${ }^{12-16}$ However, there has been controversy in the past about reports concerning dead magnetic layers and absence of magnetic order in ultrathin films on these surfaces. ${ }^{14,15}$ The fundamental key to many unresolved puzzles may be the itinerant character of TMs resulting in competing exchange interactions beyond nearest neighbors and higher-order spin interactions beyond the Heisenberg model. The latter interactions have been proposed to play a role in transition metals; however, to our knowledge, no unambiguous proof of their importance has been given.

Here, we use first-principles calculations to demonstrate that a hexagonal Fe ML can assume very different magnetic phases on a triangular lattice provided by hcp (0001) and fcc (111) surfaces of $4 d$ - and $5 d$ - transition metals, which are also experimentally accessible, e.g., $\quad \mathrm{Fe} / \mathrm{Ir}(111),{ }^{11,15}$ $\mathrm{Fe} / \mathrm{Ru}(0001),{ }^{14}$ or $\mathrm{Fe} / \mathrm{Pt}(111) .{ }^{16}$ We show that the nearestneighbor exchange interaction, $J_{1}$, in the Fe ML changes continuously from antiferromagnetic (AFM) to ferromagnetic (FM) with filling of the substrate $d$ band. Due to topological frustration on a triangular lattice, AFM coupling for Fe on $\operatorname{Re}(0001)$ and $\operatorname{Ru}(0001)$ leads to a Néel ground state with angles of $120^{\circ}$ between adjacent spins. This finding can explain unresolved experimental studies reporting the $a b$ sence of a ferromagnetic signal for $\mathrm{Fe} / \mathrm{Ru}(0001) .14,15,17,18$

For an Fe ML on substrates such as Ru, Os, Rh, or Ir, $J_{1}$ is small and interactions beyond nearest neighbors or higherorder spin interactions can be relevant. We exemplify this by studying so-called multi- $\mathbf{Q}$ states, a superposition of symmetry equivalent spin spirals, which are degenerate in the Heisenberg model but can gain energy, e.g., due to the presence of biquadratic or four-spin interactions. ${ }^{19}$ In the $4 d$-TM substrates, significant magnetic moments are induced by the Fe ML due to their high susceptibility. For Fe/Rh(111), this is a crucial effect which stabilizes an unexpected collinear ground state of ferromagnetic double-rows coupling antiferromagnetically along the $[11 \overline{2}]$ direction, a $2 \mathrm{D}$ analog of the antiferromagnetic bilayer state in Fe films on $\mathrm{Cu}(001) .{ }^{20}$ For $5 d$-TM substrates such as Ir or Re, the large spin-orbit coupling may cause a significant Dzyaloshinskii-Moriya interaction in the Fe ML which can crucially affect the magnetic order,, 10 making these systems a unique playground for complex magnetism.

We have determined the electronic and magnetic properties of $1 \mathrm{ML} \mathrm{Fe}$ on the hcp (0001) and fcc (111) surfaces of $4 d$ - and isoelectronic $5 d$-transition metals based on densityfunctional theory. Calculations have been carried out in the generalized gradient approximation (GGA) to the exchangecorrelation functional ${ }^{21}$ using the full-potential linearized augmented plane wave method, as implemented in the FLEUR code. ${ }^{22,23}$ All calculations have been performed in the scalarrelativistic approximation, i.e., neglecting the effect of spinorbit coupling.

The collinear magnetic states were investigated in systems modeled by six or seven layers of $4 d$ - or $5 d$-TM sub- 


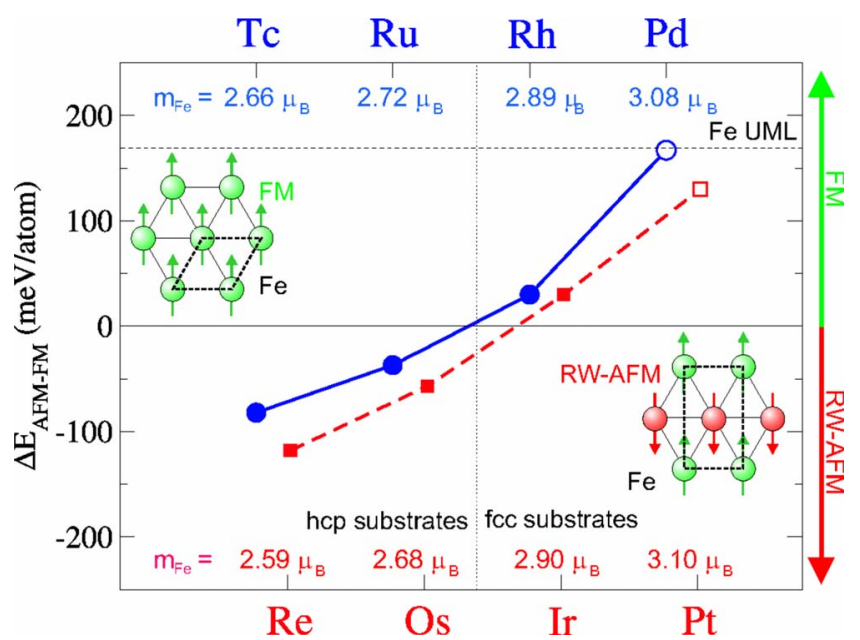

FIG. 1. (Color online) Total-energy difference between the FM $(\Delta E>0)$ and RW-AFM $(\Delta E<0)$ configurations for Fe MLs on hcp (0001) and fcc (111) surfaces of $4 d$ and $5 d$ TMs. Closed and open symbols indicate a favorable hcp or fcc stacking of the Fe ML, respectively. The magnetic moment of the Fe atoms, $m_{\mathrm{Fe}}$, is given. $\Delta E_{\mathrm{AFM}-\mathrm{FM}}$ is nearly constant for an unsupported hexagonal Fe ML with the corresponding in-plane lattice constants (dashed line).

strate with hcp or fcc stacking covered by a pseudomorphic Fe monolayer on each side of the films. We have used the experimental lattice constants which are very close to the values obtained by GGA. The structural relaxation of the Fe overlayer has been performed for both fcc and hep stackings. The noncollinear magnetic states have been studied employing an asymmetric film consisting of four substrate layers and an Fe monolayer on one side of the film at the distance optimized for the collinear (FM or AFM) state of lowest energy. For $\mathrm{Fe}$ on $\mathrm{Rh}(111)$ we have used six substrate layers; we found that adding two layers of substrate did not influence the spin-spiral dispersion by more than $6 \mathrm{meV}$. The spin spirals have been calculated exploiting the generalized Bloch theorem. ${ }^{24}$ We have used about 100 basis functions per atom for all calculations and at least $676 \mathbf{k}_{\|}$points in the twodimensional Brillouin zone (2D-BZ) for the spin-spiral calculations, $48 \mathbf{k}_{\|}$points in one quarter of the $2 \mathrm{D}$-BZ for the uudd configuration along $\overline{\Gamma-M}, 84 \mathbf{k}_{\|}$points in one half of the 2D-BZ for the uudd configuration along $\overline{\Gamma-K}$, and $32 \mathbf{k}_{\|}$ points in the $2 \mathrm{D}-\mathrm{BZ}$ for the $\mathbf{3} \mathbf{Q}$ state requiring a surface unit cell comprising of four atoms.

In order to find the magnetic ground state, we start by evaluating the total-energy difference between the FM and the row-wise AFM (RW-AFM) configuration, Fig. 1, considering hep and fcc stackings of the monolayer. Only for substrates at the end of the TM series, Pd and Pt, the Fe monolayer prefers fcc stacking. On all other substrates Fe prefers an hcp stacking. Only on Rh and Ir the energy difference between fcc and hcp stackings is sufficiently small (9.0 and $7.6 \mathrm{meV} / \mathrm{Fe}$ atom, respectively) to suggest the experimental observation of both types after film growth at room temperature. ${ }^{11,25}$

From Fig. 1 it can be concluded that Fe on substrates from the center of the TM series, Tc and Re, exhibits a clear antiferromagnetic behavior and the RW-AFM state has the low-
TABLE I. Results on the structural relaxations and magnetic moments. The in-plane lattice constant of the substrate, $a$, the interlayer distance between the Fe ML and the surface layer, $d$, the relative relaxation, $\delta d$, the distance between $\mathrm{Fe}$ atoms and substrate atoms, $d_{\mathrm{Fe}-s}$, and the magnetic moment of the Fe atoms, $m_{\mathrm{Fe}}$, are given.

\begin{tabular}{lccccc}
\hline \hline & $\begin{array}{c}a \\
\text { (a.u. })\end{array}$ & $\begin{array}{c}d \\
(\text { a.u. })\end{array}$ & $\begin{array}{c}\delta d \\
(\%)\end{array}$ & $\begin{array}{c}d_{\mathrm{Fe}-s} \\
(\text { a.u. })\end{array}$ & $\begin{array}{c}m_{\mathrm{Fe}} \\
\left(\mu_{B}\right)\end{array}$ \\
\hline $\mathrm{Fe} / \mathrm{Tc}(0001)$ & 5.17 & 3.84 & -7.4 & 4.86 & 2.66 \\
$\mathrm{Fe} / \mathrm{Ru}(0001)$ & 5.11 & 3.85 & -4.8 & 4.85 & 2.72 \\
$\mathrm{Fe} / \mathrm{Rh}(111)$ & 5.11 & 3.92 & -5.9 & 4.91 & 2.89 \\
$\mathrm{Fe} / \mathrm{Pd}(111)$ & 5.28 & 3.85 & -10.7 & 4.91 & 3.08 \\
$\mathrm{Fe} / \operatorname{Re}(0001)$ & 5.22 & 3.84 & -8.8 & 4.88 & 2.59 \\
$\mathrm{Fe} / \mathrm{Os}(0001)$ & 5.17 & 3.88 & -4.9 & 4.89 & 2.68 \\
$\mathrm{Fe} / \mathrm{Ir}(111)$ & 5.20 & 3.94 & -7.2 & 4.95 & 2.90 \\
$\mathrm{Fe} / \mathrm{Pt}(111)$ & 5.31 & 3.82 & -12.7 & 4.90 & 3.10 \\
\hline \hline
\end{tabular}

est energy. Fe on substrates from the end of the TM series is ferromagnetic. In between we observe a gradual change from a strongly AFM behavior to a strongly FM one as a function of the electron filling of the substrate. It is argued that this change in the magnetic coupling results from the $3 d-4 d$ and $3 d-5 d$ hybridization between the Fe ML and the substrate, which is altered by the $d$-band filling. ${ }^{8,26}$ This argument is supported by the fact (i) that the role of the hybridization is also apparent from the monotonous variation in the Fe magnetic moments as one moves through the TM series and (ii) the gradual change from FM to AFM coupling cannot be explained on the basis of the changing in-plane lattice constant as the comparison with unsupported MLs on the respective lattice constants shows a rather stable ferromagnetic value of about $160 \mathrm{meV} / \mathrm{Fe}$ atom, cf. Fig. 1. The in-plane lattice constants and the results on the structural relaxations are summarized in Table I. These data lead to the conclusion that the increasing magnetic moment reflects the $d$-band filling rather than different structural relaxations.

From the above results we can conclude that the antiferromagnetic exchange interaction is strong and important for $\mathrm{Fe}$ on most of these substrates except $\mathrm{Pd}$ and $\mathrm{Pt}$ on which $\mathrm{Fe}$ is clearly ferromagnetic. The antiferromagnetic interaction on a triangular lattice leads to the frustration of magnetic interactions and is the origin of complex magnetic states. $\mathrm{Fe}$ on Re or Tc exhibits strong antiferromagnetic interactions as shown by the large energy gain when assuming a RW-AFM state and the true ground state could be a $120^{\circ}$-Néel state. Due to the small energy difference between the FM and RW$\mathrm{AFM}$ order for $\mathrm{Fe}$ on $\mathrm{Os}, \mathrm{Ru}, \mathrm{Rh}$, and Ir many magnetic states have to be considered as possible ground states. Therefore, we first focus on Fe MLs on $\mathrm{Ru}(0001)$ and on $\mathrm{Rh}(111)$ as model systems of complex magnetism on a triangular lattice. $^{27}$

We study noncollinear magnetic structures by performing spin-spiral calculations. Flat spin spirals are the general solution of the classical Heisenberg model on a periodic lattice $H=-\sum_{i<j} J_{i j} \hat{\mathbf{M}}_{i} \cdot \hat{\mathbf{M}}_{j}$, where the exchange constants $J_{i j}$ determine the strength and the type of coupling between local 
(a) $120^{\circ}$ Néel state uudd state $(\bar{\Gamma}-\overline{\mathrm{K}})$ uudd state $(\bar{\Gamma}-\overline{\mathrm{M}})$
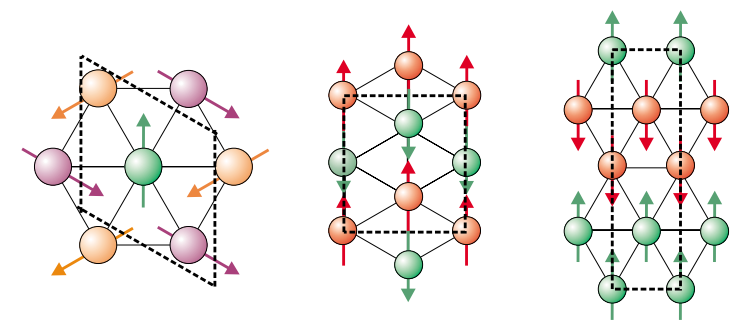

(b)

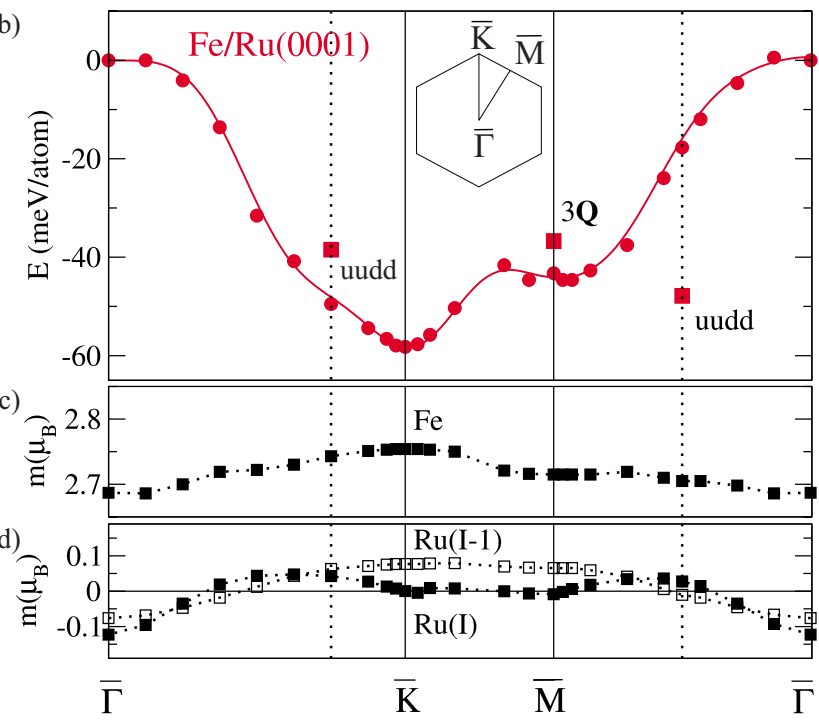

FIG. 2. (Color online) (a) Néel state and uudd states obtained from a superposition of two $90^{\circ}$ spin spirals running either along $\bar{\Gamma} \bar{K}$ or $\bar{\Gamma} \bar{M}$. (b) Total energy of spin spirals for one hep monolayer $\mathrm{Fe}$ on $\mathrm{Ru}(0001)$ (filled circles) along the high symmetry directions of the 2D-BZ (see inset). Solid line denotes a fit to the Heisenberg model up to $J_{5}$. The $3 \mathbf{Q}$ - and $u u d d$-states are included for comparison (squares). Magnetic moments of (c) Fe atoms and (d) Ru interface atoms, $\mathrm{Ru}(\mathrm{I})$ (filled squares) and subinterface atoms, $\mathrm{Ru}(\mathrm{I}-1)$ (open squares).

moments at sites $i$ and $j$ pointing along the unit vectors $\hat{\mathbf{M}}_{i}$ and $\hat{\mathbf{M}}_{j}$, respectively. Spin spirals are characterized by a wave vector $\mathbf{q}$ and the moment of an atom at site $\mathbf{R}_{i}$ is given by $\mathbf{M}_{i}\left(\mathbf{R}_{i}\right)=M\left[\cos \left(\mathbf{q} \cdot \mathbf{R}_{i}\right), \sin \left(\mathbf{q} \cdot \mathbf{R}_{i}\right), 0\right]$, where $M$ is the spin moment per atom. By considering spin spirals along the high symmetry lines of the 2D-BZ we cover an important part of the magnetic phase space. At high symmetry points, we find well-known magnetic states such as the FM state at the $\bar{\Gamma}$ point, the RW-AFM state at the $\bar{M}$ point, and the $120^{\circ}$-Néel state at the $\bar{K}$ point, cf. Fig. 2(a).

The calculated total-energy dispersion, $E(\mathbf{q})$, of spin spirals for $\mathrm{Fe} / \mathrm{Ru}(0001)$, Fig. 2(b), shows that the Néel state, i.e., for $\mathbf{q}=\bar{K}$, is the energetically most favorable magnetic configuration. The energy gain is $58 \mathrm{meV} / \mathrm{Fe}$ atom with respect to the FM state and only $13 \mathrm{meV} / \mathrm{Fe}$ atom with respect to the RW-AFM state. The magnetic moment in the Fe ML, Fig. 2(c), depends only weakly on q. Interestingly, the moments of the surface and subsurface Ru layer are of similar magnitude and change their alignment to the Fe moments, Figs. 2(c) and 2(d)
TABLE II. Heisenberg exchange constants for the hcp Fe ML on different substrates obtained by fitting the total-energy dispersion along $\bar{\Gamma}-\bar{K}-\bar{M}$ and higher order Heisenberg terms.

\begin{tabular}{lrrrrrr}
\hline \hline$(\mathrm{meV})$ & \multicolumn{1}{c}{$J_{1}$} & \multicolumn{1}{c}{$J_{2}$} & \multicolumn{1}{c}{$J_{3}$} & $J_{4}$ & \multicolumn{1}{c}{$B$} & $K$ \\
\hline $\mathrm{Fe} / \mathrm{Re}(0001)$ & -14.5 & -0.5 & -5.4 & -0.5 & 3.1 & 1.8 \\
$\mathrm{Fe} / \mathrm{Ru}(0001)$ & -6.4 & 0.7 & -0.3 & 0.4 & -0.6 & 1.1 \\
$\mathrm{Fe} / \mathrm{Rh}(111)$ & 3.8 & -0.6 & -1.6 & 0.3 & -0.6 & 0.0 \\
$\mathrm{Fe} / \mathrm{Ir}(111)$ & 4.2 & -0.8 & 0.3 & 0.2 & 0.7 & 0.4 \\
\hline \hline
\end{tabular}

To interpret our calculations, we have mapped the results onto a 2D Heisenberg model which allows us to determine effective exchange constants $J_{i j}$ between moments of the $\mathrm{Fe}$ atoms in the ML. Figure 2(b) shows that a good fit is obtained by including up to five nearest neighbors. From the $J_{i j}$ 's, given in Table II, we conclude that the main effect of the substrate is to change the nearest-neighbor exchange coupling, $J_{1}$, from FM $\left(J_{1}>0\right)$ for Rh and Ir to $\operatorname{AFM}\left(J_{1}<0\right)$ for $\mathrm{Ru}$ and Re.

For itinerant magnets such as iron, it is not a priori clear that the Heisenberg model, which relies on localized magnetic moments, can provide a good description. The next higher-order terms beyond the Heisenberg model which occur in a perturbative expansion of the Hubbard mode ${ }^{28}$ are the biquadratic and four-spin interactions. These terms can lead to energy contributions on the order of $15 \mathrm{meV} / \mathrm{Fe}$ atom $^{19}$ which is similar to the energy difference between Néel and RW-AFM state of $\mathrm{Fe} / \mathrm{Ru}(0001)$.

In order to find the magnitude of these interactions, we consider linear combinations of spin spirals, the multi-Q states. The degeneracy of single- and multi-Q states within the Heisenberg model is lifted by higher-order interactions. By calculating the energy differences between suitable single-Q and multi-Q states, we can obtain values for the nearest-neighbor biquadratic interaction, $B$, and four-spin interaction, $K$. One pair of such states are spin spirals at the $\bar{M}$ points of the $2 \mathrm{D}-\mathrm{BZ}$ and the $3 \mathbf{Q}$ state constructed from the three independent $\bar{M}$ points. ${ }^{19}$ As a second pair we choose the spin spiral at $\mathbf{Q}_{3 \bar{K} / 4}$ and a superposition of two such spirals with opposite rotation sense, a so-called uudd state [middle panel of Fig. 2(a)]. The total-energy differences are

$$
\begin{gathered}
E_{3 \mathrm{Q}}-E_{\mathrm{RW}-\mathrm{AFM}}=(16 / 3)\{2 K+B\}, \\
E_{\text {uudd }}-E_{3 \bar{K} / 4}=4\{2 K-B\},
\end{gathered}
$$

marked by squares in Fig. 2(b) and the obtained constants $B$ and $K$ are given in Table II.

The large energy differences between the multi- $\mathbf{Q}$ and single-Q states of 7 and $11 \mathrm{meV} / \mathrm{Fe}$ atom for the $3 \mathbf{Q}$ - and the uudd states, respectively, found for $\mathrm{Fe} / \mathrm{Ru}(0001)$ clearly demonstrate its itinerant character. The extracted values for the biquadratic and four-spin interaction are of the order of the exchange constants beyond nearest neighbors and cannot be neglected in finding the ground state. Higher-order interactions play a similar role for other substrates, as shown in Table II. 


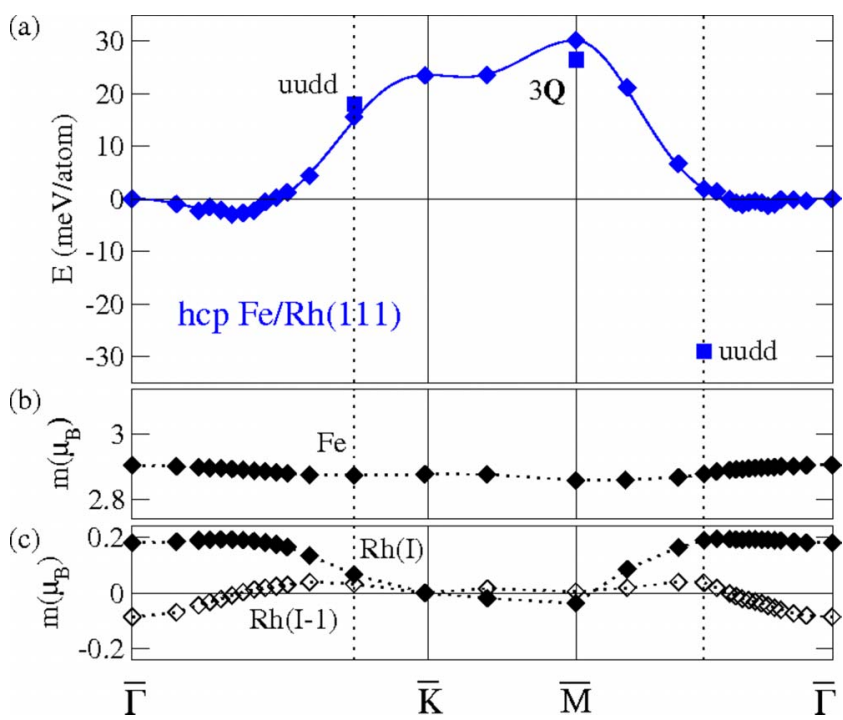

FIG. 3. (Color online) (a) Total energy of one hcp monolayer Fe on $\mathrm{Rh}(111)$ for spin spirals along high-symmetry lines (cf. Fig. 2) and multi-Q states. Solid line denotes a fit to the Heisenberg model up to $J_{5}$. Magnetic moments of the Fe atoms and the $\mathrm{Rh}$ interface atoms $[\mathrm{Rh}(\mathrm{I})$, open symbols $]$ and subinterface atoms $[\mathrm{Rh}(\mathrm{I}-1)$, filled symbols] are shown in panels (b) and (c), respectively.

The Fe ML also induces considerable magnetic moments in the Ru substrate due to its high susceptibility. The induced magnetic moments of $\mathrm{Ru}$ depend on the magnetic structure in the Fe ML and their size contributes to the total energy of the system. This effect can be dramatic as seen by comparing the uudd state along $\bar{\Gamma}-\bar{M}$ and $\bar{\Gamma}-\bar{K}$, cf. right panel of Fig. $2(\mathrm{a})$, to the corresponding single- $\mathbf{Q}$ states. The energy difference due to biquadratic and four-spin interactions should be the same in both cases, which is clearly not the case. This finding can be traced back to the difference in the induced magnetic moments in the substrate for the two uudd states.

We now turn to $\mathrm{Fe}$ on $\mathrm{Rh}$ (111) for which the FM state was slightly favorable among the collinear states considered in Fig. 1. Our spin-spiral calculations, however, indicate a noncollinear ground state with a spiral vector $\mathbf{q}$ of about 0.225 $\times \frac{2 \pi}{a}$ along $\bar{\Gamma}-\bar{K}$, about $2.5 \mathrm{meV} / \mathrm{Fe}$ atom below the FM state (Fig. 3). From the fitting to the Heisenberg model, cf. Table II, we indeed find a very small FM nearest-neighbor exchange constant while second- and third-nearest neighbors prefer an AFM alignment of a magnitude comparable to the FM exchange.

Including the multi-Q states in our search for the groundstate results in the uudd solution $(\bar{\Gamma}-\bar{M})$ as the most favorable configuration with a large energy gain of $29.0 \mathrm{meV} / \mathrm{Fe}$ atom with respect to the single-Q $\mathbf{Q}$ state. However, the real magnetic ground state might be even more complex due to the competing interactions involved making $\mathrm{Fe} / \mathrm{Rh}(111)$ a truly intriguing system and a challenge for an experimental investigation.

In the phase diagram of the 2D Heisenberg model, shown
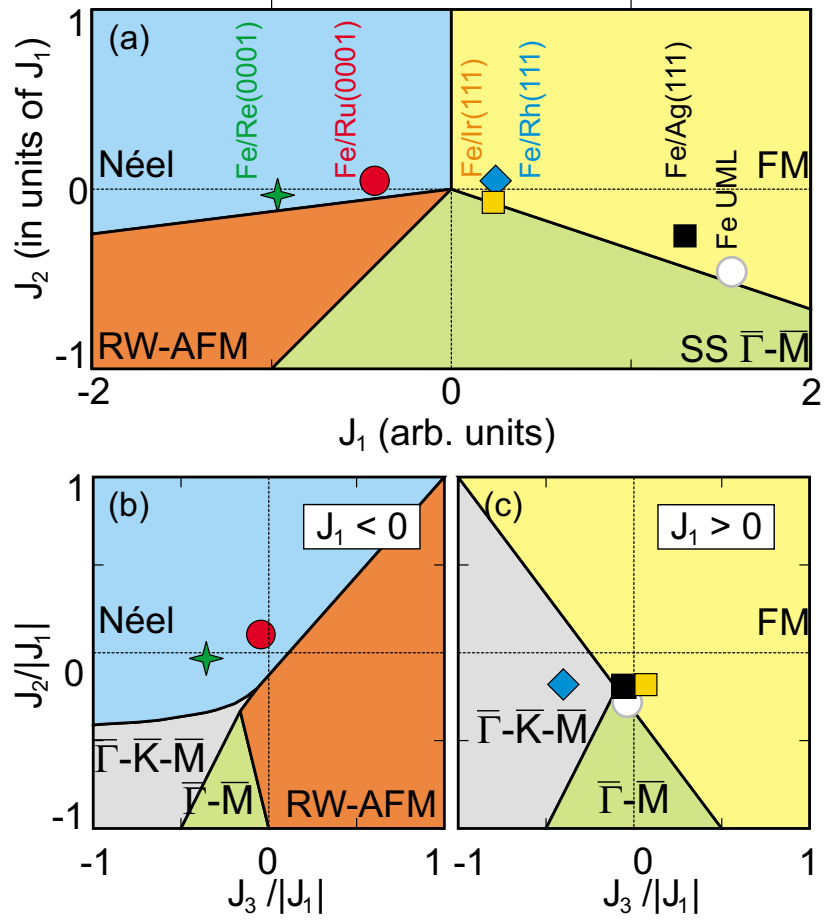

FIG. 4. (Color online) Phase diagrams of the classical Heisenberg model for a 2D hexagonal lattice for states on the highsymmetry lines. (a) $J_{1}-J_{2}$ plane for $J_{3}=0 . J_{2}-J_{3}$ plane for (b) $J_{1}$ $>0$ and (c) $J_{1}<0$. (Filled symbols denote values obtained from fits for the Fe ML on $4 d$ - and $5 d$-TM substrates.)

in Fig. 4, we can provide a complete picture of the substrates' impact on the $\mathrm{Fe}$ exchange coupling by including spin spiral calculations for an Fe ML on $\operatorname{Re}(0001), \operatorname{Ir}(111)$, and $\mathrm{Ag}(111)$. In the $J_{1}-J_{2}$ plane of the diagram, Fig. 4(a), we see that the $d$-band filling of the substrate drives the system along the line $J_{2} \approx 0$ from a Néel configuration on $\mathrm{Re}$ and $\mathrm{Ru}$ to the FM solution on Ir and Rh. For small $J_{1}$, we need to consider also the phase diagrams in the $J_{2}-J_{3}$ plane showing the spin spiral minimum of $\mathrm{Fe}$ on $\mathrm{Rh}(111)$ in the $\overline{\Gamma K M}$ direction, Fig. 4(c), and the $120^{\circ}$-Néel state of $\operatorname{Re}(0001)$, Fig. 4(b). In contrast, the FM state is the most favorable single- $\mathbf{Q}$ state for the hcp Fe ML on $\operatorname{Ir}(111) .{ }^{27}$

In conclusion, we have proposed Fe MLs on hexagonal surfaces of late $4 d$ and $5 d$ TMs as promising systems to study experimentally the magnetic interactions in TMs, e.g., by proving the influence of spin interactions beyond the Heisenberg model. Alloying of the substrate, e.g., PtRu(0001), ${ }^{29}$ may allow an additional fine tuning of the degree of disorder in 2D systems as proposed in Ref. 26.

Financial support of the Stifterverband für die Deutsche Wissenschaft, the Interdisciplinary Nanoscience Center Hamburg, and the ESF EUROCORES Programme SONS under Contract No. ERAS-CT-2003-980409 is gratefully acknowledged. 
*Corresponding author; heinze@ @ physnet.uni-hamburg.de

${ }^{1}$ C. Chappert, A. Fert, and F. Nguyen van Dau, Nature Mater. 6, 813 (2007).

${ }^{2}$ P. Gambardella, A. Dallmeyer, K. Maiti, M. C. Malagoli, W. Eberhardt, K. Kern, and C. Carbone, Nature (London) 416, 301 (2002).

${ }^{3}$ C. F. Hirjibehedin, C. P. Lutz, and A. J. Heinrich, Science 312, 1021 (2006)

${ }^{4}$ S. Lounis, P. H. Dederichs, and S. Blügel, Phys. Rev. Lett. 101, 107204 (2008).

${ }^{5}$ J. Honolka, K. Kuhnke, L. Vitali, A. Enders, K. Kern, S. Gardonio, C. Carbone, S. R. Krishnakumar, P. Bencok, S. Stepanow, and P. Gambardella, Phys. Rev. B 76, 144412 (2007).

${ }^{6}$ N. Néel, J. Kröger, R. Berndt, T. O. Wehling, A. I. Lichtenstein, and M. I. Katsnelson, Phys. Rev. Lett. 101, 266803 (2008).

${ }^{7}$ C. Iacovita, M. V. Rastei, B. W. Heinrich, T. Brumme, J. Kortus, L. Limot, and J. P. Bucher, Phys. Rev. Lett. 101, 116602 (2008).

${ }^{8}$ A. Kubetzka, P. Ferriani, M. Bode, S. Heinze, G. Bihlmayer, K. von Bergmann, O. Pietzsch, S. Blügel, and R. Wiesendanger, Phys. Rev. Lett. 94, 087204 (2005).

${ }^{9}$ M. Bode, M. Heide, K. von Bergmann, P. Ferriani, S. Heinze, G. Bihlmayer, A. Kubetzka, O. Pietzsch, S. Blügel, and R. Wiesendanger, Nature (London) 447, 190 (2007).

${ }^{10}$ P. Ferriani, K. von Bergmann, E. Y. Vedmedenko, S. Heinze, M. Bode, M. Heide, G. Bihlmayer, S. Blügel, and R. Wiesendanger, Phys. Rev. Lett. 101, 027201 (2008).

${ }^{11}$ K. von Bergmann, S. Heinze, M. Bode, E. Y. Vedmedenko, G. Bihlmayer, S. Blügel, and R. Wiesendanger, Phys. Rev. Lett. 96, 167203 (2006).

${ }^{12}$ M. Albrecht, M. Maret, J. Köhler, B. Gilles, R. Poinsot, J. L. Hazemann, J. M. Tonnerre, C. Teodorescu, and E. Bucher, Phys. Rev. Lett. 85, 5344 (2000).
${ }^{13}$ F. El Gabaly, S. Gallego, C. Muñoz, L. Szunyogh, P. Weinberger, C. Klein, A. K. Schmid, K. F. McCarty, and J. de la Figuera, Phys. Rev. Lett. 96, 147202 (2006).

${ }^{14}$ C. Liu and S. D. Bader, Phys. Rev. B 41, 553 (1990).

${ }^{15}$ S. Andrieu, M. Piecuch, and J. F. Bobo, Phys. Rev. B 46, 4909 (1992).

${ }^{16}$ D. Repetto, T. Y. Lee, S. Rusponi, J. Honolka, K. Kuhnke, V. Sessi, U. Starke, H. Brune, P. Gambardella, C. Carbone, A. Enders, and K. Kern, Phys. Rev. B 74, 054408 (2006).

${ }^{17}$ E. Bauer, J. Phys. Cond. Mat. 11, 9365 (1999).

${ }^{18}$ D. Tian, H. Li, F. Jona, and P. M. Marcus, Solid State Commun. 80, 783 (1991).

${ }^{19} \mathrm{Ph}$. Kurz, G. Bihlmayer, K. Hirai, and S. Blügel, Phys. Rev. Lett. 86, 1106 (2001).

${ }^{20}$ T. Asada and S. Blügel, Phys. Rev. Lett. 79, 507 (1997).

${ }^{21}$ Y. Zhang and W. Yang, Phys. Rev. Lett. 80, 890 (1998).

${ }^{22} \mathrm{http} / / / \mathrm{www}$.flapw.de

${ }^{23} \mathrm{Ph}$. Kurz, F. Forster, L. Nordström, G. Bihlmayer, and S. Blügel, Phys. Rev. B 69, 024415 (2004).

${ }^{24}$ C. Herring, in Magnetism, edited by G. Rado and H. Suhl (Academic, New York, 1966).

${ }^{25}$ K. von Bergmann, S. Heinze, M. Bode, G. Bihlmayer, S. Blügel, and R. Wiesendanger, N. J. Phys. 9, 396 (2007).

${ }^{26}$ P. Ferriani, I. Turek, S. Heinze, G. Bihlmayer, and S. Blügel, Phys. Rev. Lett. 99, 187203 (2007).

${ }^{27}$ For the fcc Fe ML on $\operatorname{Ir}(111)$ a complex nanoscale magnetic structure has been observed and is attributed to higher-order spin interactions (Ref. 11).

${ }^{28}$ A. H. MacDonald, S. M. Girvin, and D. Yoshioka, Phys. Rev. B 37, 9753 (1988).

${ }^{29}$ H. E. Hoster, A. Bergbreiter, P. M. Erne, T. Hager, H. Rauscher, and R. J. Behm, Phys. Chem. Chem. Phys. 10, 3812 (2008). 